\title{
Cardiovascular fitness in males at age 18 and risk of serious depression in adulthood: Swedish prospective population-based study ${ }^{\dagger}$
}

Maria A. I. Åberg, Margda Waern, Jenny Nyberg, Nancy L. Pedersen, Ylva Bergh, N. David Åberg, Michael Nilsson, H. Georg Kuhn and Kjell Torén

\section{Background}

Studies suggest a role for cardiovascular fitness in the prevention of affective disorders.

\section{Aims \\ To determine whether cardiovascular fitness at age 18 is associated with future risk of serious affective illness.}

\section{Method}

Population-based Swedish cohort study of male conscripts $(n=1117292)$ born in 1950-1987 with no history of mental illness who were followed for 3-40 years. Data on cardiovascular fitness at conscription were linked with national hospital registers to calculate future risk of depression (requiring in-patient care) and bipolar disorder

\section{Results}

In fully adjusted models low cardiovascular fitness was associated with increased risk for serious depression (hazard ratios $(H R)=1.96,95 \%, \mathrm{Cl} 1.71-2.23)$. No such association could be shown for bipolar disorder $(H R=1.11$, 95\% Cl 0.84-1.47).

\section{Conclusions}

Lower cardiovascular fitness at age 18 was associated with increased risk of serious depression in adulthood. These results strengthen the theory of a cardiovascular contribution to the aetiology of depression.

\section{Declaration of interest}

None.
Intervention studies suggest that physical exercise may improve cognition and mood in people with an already established depression. ${ }^{1,2}$ A proposed mechanism of action is that physical exercise can lead to a reversal of the reduced neuronal plasticity that is observed in both depression and bipolar disorders. ${ }^{3-5}$ However, the role of cardiovascular fitness in the prevention of these disorders remains unclear. Several cross-sectional epidemiological studies have documented an association between self-reported sedentary lifestyle and depression. ${ }^{6,7}$ The majority of studies have focused on adults, but similar trends have also emerged in studies of adolescents. ${ }^{8,9}$ Prospective studies are relatively few and follow-up times are in most cases limited. ${ }^{10-12}$ Regarding studies that focus specifically on physical fitness in adolescence and young adulthood, results are inconsistent. In a cohort study of 12- to 15-year-old pupils living in Norway $(n=2464)$ who were followed for 1 year, low levels of vigorous exercise and high levels of sedentary activities constituted independent risk factors for the development of depressive symptoms in boys but not in girls. ${ }^{8}$ A prospective study from London of 2789 pupils aged 11-14 found no evidence for an association between a change in physical activity and depressive symptoms at 2-year follow-up. ${ }^{13}$ Sample size is often a limitation in this type of study as depression is an outcome influenced by multiple factors and studies often lack sufficient power to elucidate the role of moderating and mediating variables. ${ }^{14}$ Moreover, most previous studies have relied primarily on questionnaires or self-rating scales for the measurement of physical activity. There is a need for large studies with long follow-up times and objective measures of physical performance.

We performed a prospective cohort study of all Swedish men born in 1950-1987 who were enlisted for mandatory military service at age $18(n=1117292)$ and followed for at least 3 but up to 40 years. The aim was to determine whether cardiovascular fitness measured during ergometer cycling at conscription was

TSee editorial, pp. 337-338, this issue. associated with the risk of developing serious affective disorders. We wanted to determine whether such an association might also remain after adjustment for familial influences. As previous studies indicate that cardiovascular aspects of fitness, rather than muscle strength per se, could be the underlying mechanism linking physical exercise and cognitive function, ${ }^{15,16}$ an additional hypothesis was that an association between cardiovascular fitness and later risk of affective disorders would be independent of muscle strength.

\section{Method}

\section{Study population}

A cohort of 18-year-old Swedish males who enlisted for military service between 1968 and 2005 (i.e. born between 1950 and 1987, $n=1353723$ ) was compiled from the Swedish Military Service Conscription Register. During that time Swedish law required all 18-year-old Swedish men to enlist. Exemptions were granted only for incarcerated males, severe chronic medical or mental conditions or handicaps documented by a medical certificate (approximately $2-3 \%$ of the yearly male population). When enlisting, males underwent extensive and highly standardised physical and psychological examinations by medical doctors and psychologists prior to conscription assignment in the Swedish armed forces. Individuals with any history of or ongoing psychiatric disorders or symptoms at the examinations were excluded from the current study. The ethics committee of University of Gothenburg and confidentiality clearance at Statistics Sweden approved the study.

\section{Swedish Military Service Conscription Register data}

\section{Cardiovascular fitness test}

Cardiovascular fitness was assessed using the cycle ergonometric test. The procedure, including elements of validity and reliability 
has been described in detail previously. ${ }^{17}$ Briefly, after a normal resting electrocardiogram (ECG), 5 min of submaximal exercise was performed at work rates of 75-175 W, depending on body mass. The work rate was continuously increased by $25 \mathrm{~W} / \mathrm{min}$ until volitional exhaustion. The individual was instructed to maintain pedal cadence between $60-70 \mathrm{rpm}$. Heart rate was continuously measured. The final work rate (Wmax) was recorded and divided by body mass. Wmax/kg was employed, because of better correlation with measured maximum oxygen consumption $\left(\mathrm{VO}_{2 \max }\right)$ (correlation coefficient $\sim 0.9$ ) than predicted $\mathrm{VO}_{2 \max }$ (correlation coefficient $\sim 0.6-0.7){ }^{18,19}$ The resulting value (Wmax $/ \mathrm{kg}$ ) was transformed into stanine scores, with 1 as the lowest and 9 as the maximal performance, and served as a measure of cardiovascular fitness.

\section{Muscular strength test}

Isometric muscle strength was measured by knee extension (weighted 1.3), elbow flexion (weighted 0.8), and hand grip (tested with a tensiometer; weighted 1.7). ${ }^{17}$ Weighted values were integrated into one estimate in kilopond (before 1979) or Newton (after 1979) and divided into nine stanines.

\section{Diagnoses recorded at conscription}

Men who fulfilled criteria for previous or ongoing psychiatric disorders or symptoms (ICD-8 and ICD-9 codes 290-319; ICD-10 codes F00-99; all mental and behavioural disorders), ${ }^{20-22}$ diagnosed by psychologists and medical doctors, at conscription were excluded from the analyses $(n=77028)$.

\section{Register linkage}

All Swedes have a unique personal identification number making linkage to other registers possible. Links to the Multi-Generation Register (www.scb.se/Pages/List_257501.aspx) enabled the identification of full brothers. Education and occupation information was obtained from the longitudinal integration database for health insurance and labour market studies (Swedish acronym LISA). Psychiatric diagnoses were obtained from the Swedish National Hospital Discharge Register at the National Board of Health and Welfare.

LISA

The LISA database (www.scb.se/Pages/List_257743.aspx) was initiated in 1990 and includes all registered residents aged 16 years and older. The database, which is annually updated, integrates data from the labour market, as well as educational and social sectors. Information on parental education was obtained from LISA and was rated in seven levels: pre-high school education less than 9 years, pre-high school education 9 years, high school education, college education (less than 2 years), college education ( 2 or more years), postgraduate education and postgraduate training.

\section{In-patient episodes}

The Swedish National Hospital Discharge Register covers virtually all in-patient care for psychiatric disorders since 1964. Discharge diagnoses are coded by the physician in accordance with the ICD, versions 8,9 or 10 . We identified diagnostics codes for first onset of affective disorders from 1969 to 31 December 2008. The specific ICD-8, ICD-9 and ICD-10 codes that were used in the analyses are shown in online Table DS1. For the purpose of this study, we use the term serious depression to denote the (non-bipolar) depressions identified by this procedure. People who initially received a discharge diagnosis of depression but who were later admitted to hospital with manic/hypomanic episodes $(n=12)$ were included in the bipolar group only. Separate analyses were performed excluding individuals with any diseases of the circulatory system (ICD-8 and ICD-9 codes 390-459; ICD-10 codes 100-199) preceding or coinciding with the first onset of depression.

\section{Statistical analyses}

All statistical calculations were performed with SAS version 8.1 on Windows XP. We used Cox proportional hazards models to assess the influence of the cardiovascular performance at age 18 on the occurrence of affective diagnoses later in life. Cardiovascular fitness and muscle strength were categorised as low (stanine score 1-3), medium (stanine score 4-6) and high (stanine score 7-9); the high group was the reference category.

Age was used as the time axis, thus controlling for age. To assess effects of secular variation in diagnosis rate and differences in conscription procedures over calendar time, we adjusted for calendar years by stratifying the Cox model by decade (1960s, 1970 s, etc.). The follow-up period began at the date of conscription (baseline) and ended on 31 December 2008 (minimum 3 years and maximum 40 years follow-up). Separate analyses were also performed excluding individuals who emigrated or died during the follow-up period.

To reduce the risk for possible reverse causation - i.e. early symptoms at the time of conscription, for example depression affecting performance on the physical tests - we restricted the analysis to men with no history of psychiatric diagnoses or symptoms as well as those with no previous registered in-patient affective disorder diagnoses. To further reduce baseline misclassification, we performed separate analyses excluding incident 'cases' in the first year.

Because differences among regions and test centres could introduce bias, geographical region and conscription test centres were considered as possible confounders and were adjusted for. Some studies have reported higher prevalence of obesity in patients with affective disorders and recent studies suggest that body mass index (BMI) is associated with the onset, prognosis and outcome of affective disorders. ${ }^{23,24}$ Therefore adjustment for BMI and also separate analyses among subgroups (BMI $<25$ and $\mathrm{BMI} \geqslant 25$ ) were performed. Parental educational level was included as a confounder. Separate analyses were also performed among subgroups of parental education level (pre-high school, high school and university).

At the time that the ICD- 8 was in use, clinicians often classified endogenous depression as 296.20 (manic depressive psychosis, depressed type), even in the absence of a history of manic episode. This type of episode would come to be classified as unipolar affective psychosis, melancholia (296B) in ICD-9 and as major depression in ICD-10. The analyses regarding bipolar disorder were therefore redone after excluding people with an ICD-8 diagnosis of manic depressive psychosis, depressed type (296.20) when evidence of at least one manic/hypomanic episode was lacking.

To further assess potential effects of familial factors, subanalyses were performed within full-brother pairs of conscripts. We used the Cox's proportional hazards model adjusting for the above listed confounders but with the brother's/brothers' diagnosis/diagnoses as an additional explanatory variable. Separate Cox proportional hazards models were created for each muscle strength category. The fact that the study spans over a long time could have had an impact on our results as a result of latency of effect. To study how follow-up time affected the associations, 
separate analyses were performed with the follow-up times 1-10, $11-20,21-30$ and $31-40$ years.

\section{Results}

Our analyses are based on the 1117292 conscripts with complete information on physical performance, geographical region, conscription year, conscription test centre, parental educational level and BMI. During the follow-up period 1969-2008 the numbers of individuals who received in-patient treatment for depression was 12427 and for bipolar disorder 3626 corresponding to $1.11 \%$ and $0.32 \%$ of the male Swedish population between ages 18 and 58 (Table 1). The mean time from assessment of cardiovascular fitness at age 18 to the first in-patient admission was 16.7 years for depression and 14.1 years for bipolar disorder. The distribution over time between assessment and first admission is shown in Table 2 .

\section{Cardiovascular fitness and muscle strength at age 18 and future serious affective disorder}

Cardiovascular fitness and muscle strength scores were transformed to stanines that were then collapsed into three categories (low, medium and high) (Table 3). Poor performance on the cardiovascular fitness tests, as measured by Wmax $/ \mathrm{kg}$ using an ergometer cycle at age 18, was associated with an increased risk of being admitted to hospital with depression later in life (Table 3). We found an inverse relationship with highest hazard ratios

Table 1 Numbers of male conscripts admitted to hospital with depression and bipolar disorder by level of cardiovascular fitness at age $18^{\mathrm{a}}$

\begin{tabular}{|lrcr|}
\hline $\begin{array}{l}\text { Cardiovascular } \\
\text { fitness }\end{array}$ & $\begin{array}{c}\text { Conscripts } \\
n\end{array}$ & $\begin{array}{c}\text { Depression } \\
n(\%)\end{array}$ & $\begin{array}{c}\text { Bipolar disorder } \\
n(\%)\end{array}$ \\
\hline 1 & 836 & $17(2.03)$ & $1(0.12)$ \\
\hline 2 & 4634 & $94(2.03)$ & $22(0.47)$ \\
\hline 3 & 34008 & $670(1.97)$ & $167(0.49)$ \\
\hline 4 & 103572 & $1582(1.53)$ & $358(0.35)$ \\
\hline 5 & 178885 & $2420(1.35)$ & $576(0.32)$ \\
\hline 6 & 311893 & $3105(1.00)$ & $825(0.26)$ \\
\hline 7 & 165235 & $1666(1.01)$ & $530(0.32)$ \\
\hline 8 & 114707 & $956(0.83)$ & $310(0.27)$ \\
\hline 9 & 203522 & $1917(0.94)$ & $837(0.41)$ \\
\hline All levels & 1117292 & 12427 & 3626 \\
\hline $\begin{array}{l}\text { a. Assessed by cycle ergonometry. Stanine scores: cardiovascular fitness score } \\
\text { of 9 indicates maximal performance. }\end{array}$ & & \\
\hline
\end{tabular}

Table 2 Numbers of male conscripts admitted to hospital with depression and bipolar disorder by year of first admission

\begin{tabular}{|lcc|}
\hline $\begin{array}{l}\text { Years to first } \\
\text { admission }\end{array}$ & $\begin{array}{c}\text { Depression } \\
n(\%)\end{array}$ & $\begin{array}{c}\text { Bipolar disorder } \\
n(\%)\end{array}$ \\
\hline $0-4$ & $1361(10.95)$ & $571(15.75)$ \\
\hline $5-9$ & $1966(15.82)$ & $761(20.99)$ \\
\hline $10-14$ & $2105(16.94)$ & $743(20.49)$ \\
\hline $15-19$ & $2096(16.87)$ & $572(15.77)$ \\
\hline $20-24$ & $1967(15.83)$ & $399(11.00)$ \\
\hline $25-29$ & $1614(12.99)$ & $297(8.19)$ \\
\hline $30-34$ & $1019(8.20)$ & $212(5.85)$ \\
\hline $35-39$ & $299(2.41)$ & $71(1.96)$ \\
\hline All levels & 12427 & 3626 \\
\hline
\end{tabular}

for depression in the low fitness group (stanine score 1-3). The strength of the associations changed little in models that controlled for calendar year, conscription test centre, region, BMI and parental education. Due to the large number of observations, the $P$-values were very small (in all analyses when testing if the 95\% confidence interval was separated from 1 the $P$-values were $<0.0001)$. Therefore $P$-values were not reported and the risk for Type-I errors was considered to be very low.

Belonging to the low fitness group compared with the high fitness group was associated with a moderately increased risk of bipolar disorder later in life. In contrast, belonging to the medium group compared with the high group was associated with a small but significantly reduced risk of bipolar disorder. Excluding the 197 individuals with diagnosis coded 296.20 from the bipolar group (see Method) did not affect the hazard ratios. Separate analyses, which excluded individuals who emigrated $(n=54493)$ or died ( $n=13014)$ during the follow-up period, yielded similar results for both depression and bipolar disorder (data not shown). Separate analyses with a latency time for diagnosis of at least 1 year after conscription examination did not affect the hazard ratios. In order to determine whether the associations differed with regard to BMI, the study sample was stratified into two groups: BMI $<25$ (under and normal weight) and BMI $\geqslant 25$ (overweight). The associations remained unchanged. Subgroup analyses of parental educational level (pre-high school, high school and university) revealed no differential association. Separate analyses excluding individuals with circulatory system disease preceding or coinciding with the first onset of depression $(n=1020)$ or bipolar disorder $(n=163)$ did not affect the hazard ratios. For muscle strength, there was a dose-response relation with the highest hazard ratios for depression in the low muscle strength group. Only the lowest scores of muscle strength were significantly associated with later risk of bipolar disorder (Table 3).

\section{Impact of familial influences on cardiovascular fitness - affective disorder associations}

In the total cohort there were 389474 participants with one or more full brothers. To adjust for familial factors in associations between cardiovascular fitness and later risk of serious affective disorder, separate subanalyses were performed by including the full brother's/brothers' diagnosis/diagnoses as an additional explanatory variable (Table 4). Although having one or more brothers with depression was associated with a threefold risk of future depression, cardiovascular fitness remained a significant predictor in the model. Having one or more brothers with bipolar disorder was associated with a ninefold risk of developing the disorder. Low cardiovascular fitness did not predict bipolar disorder in this model. Excluding the individuals with the diagnosis coded 296.20 from the bipolar group did not affect the hazard ratios. Results remained also after exclusion of men who emigrated $(n=18898)$ or died $(n=4223)$ during the follow-up period. Separate analyses with a latency time for diagnosis of at least 1 year did not affect the hazard ratios.

\section{Influence of muscle strength on cardiovascular fitness - depression associations}

As we found no clear evidence of an association between cardiovascular fitness and bipolar disorder (Table 4), the following analyses were performed for unipolar depression only.

One hypothesis for the present study was that cardiovascular fitness, more than muscular strength, would be associated with a higher later risk for affective disorders. An inverse relationship with highest hazard ratios for serious depression was observed 
Table 3 Hazard ratios for depression and bipolar disorder in relation to cardiovascular fitness and muscle strength test score in a national cohort of 18 -year-old male conscripts $(n=1117292)$ with age-adjusted and fully adjusted models

\begin{tabular}{|c|c|c|c|c|}
\hline & \multirow[b]{2}{*}{$n$} & \multicolumn{3}{|c|}{ Hazard ratio $(95 \% \mathrm{Cl})$} \\
\hline & & Age adjusted & Adjusted $^{a}$ & Adjusted $^{b}$ \\
\hline Depression & 12427 & & & \\
\hline \multicolumn{5}{|l|}{ Cardiovascular fitness $^{c}$} \\
\hline Low & 39478 & $1.86(1.73-2.01)$ & $1.82(1.68-1.96)$ & $1.80(1.64-1.99)$ \\
\hline Medium & 594350 & $1.38(1.33-1.43)$ & $1.33(1.28-1.39)$ & $1.30(1.24-1.37)$ \\
\hline \multicolumn{5}{|l|}{ Muscle strength $^{d}$} \\
\hline LoW & 118769 & $1.48(1.40-1.57)$ & $1.47(1.38-1.60)$ & $1.43(1.32-1.55)$ \\
\hline Medium & 643924 & $1.13(1.08-1.18)$ & $1.13(1.08-1.18)$ & $1.11(1.05-1.17)$ \\
\hline Bipolar disorder & 3626 & & & \\
\hline \multicolumn{5}{|l|}{ Cardiovascular fitness $^{\mathrm{C}}$} \\
\hline Low & 39478 & $1.24(1.07-1.44)$ & $1.19(1.02-1.38)$ & $1.36(1.13-1.64)$ \\
\hline Medium & 594350 & $0.89(0.83-0.95)$ & $0.90(0.84-0.96)$ & $0.88(0.81-0.96)$ \\
\hline \multicolumn{5}{|l|}{ Muscle strength ${ }^{d}$} \\
\hline LoW & 118769 & $1.29(1.15-1.44)$ & $1.21(1.07-1.36)$ & $1.21(1.04-1.40)$ \\
\hline Medium & 643924 & $1.11(1.03-1.20)$ & $1.06(0.98-1.15)$ & $1.01(0.92-1.11)$ \\
\hline $\begin{array}{l}\text { a. Adjusted for calendar yea } \\
\text { b. Adjusted for calendar yea } \\
\text { c. Reference category: high } \\
\text { d. Reference category: high }\end{array}$ & t centre, pa & & & \\
\hline
\end{tabular}

for both cardiovascular fitness and muscle strength, although the association was stronger for cardiovascular fitness (Table 3). We also expected that any association between cardiovascular fitness and later risk of serious affective disorders should be independent of muscle strength. Therefore, we examined the influence of muscular strength on the associations cardiovascular fitness/ depression, by conducting analyses in which the cohort was stratified regarding muscle strength. Low and medium (compared with high) cardiovascular fitness was associated with an increased risk of future serious depression when stratified for low, medium and high muscle strength (Table 5). Conversely, we also examined the influence of cardiovascular fitness on the associations muscular strength/depression. Low and medium (compared with high) muscular strength was not associated with an increased risk of future serious depression when stratifying for low cardiovascular fitness and only low muscle strength was associated with an increased risk of future serious depression when stratifying for medium and high cardiovascular fitness (Table 6).

Finally, in a multivariate model adjusting for calendar year, $\mathrm{BMI}$, region, conscription test centre, parental education and using muscle strength as a covariate, the hazard ratios for severe

Table 4 Subanalyses for conscripts $(n=389474)$ with one or more brothers: hazard ratios for serious depression and bipolar disorder in relation to cardiovascular fitness test score at age 18

\begin{tabular}{|c|c|c|c|}
\hline & \multirow[b]{2}{*}{$n$} & \multicolumn{2}{|c|}{ Hazard ratio $(95 \% \mathrm{Cl})$} \\
\hline & & Age adjusted & Adjusted $^{a}$ \\
\hline Depression & \multicolumn{3}{|l|}{4117} \\
\hline \multicolumn{4}{|l|}{ Cardiovascular fitness } \\
\hline Low $^{b}$ & & $2.05(1.80-2.33)$ & $1.96(1.71-2.23)$ \\
\hline Medium $^{b}$ & & $1.39(1.30-1.48)$ & $1.33(1.25-1.42)$ \\
\hline Brother $^{\mathrm{c}}$ & & $2.87(2.47-3.33)$ & $2.92(2.51-3.40)$ \\
\hline Bipolar disorder & \multicolumn{3}{|l|}{1238} \\
\hline \multicolumn{4}{|l|}{ Cardiovascular fitness } \\
\hline Low $^{b}$ & & $1.19(0.91-1.56)$ & $1.11(0.84-1.47)$ \\
\hline Medium $^{b}$ & & $0.89(0.79-1.00)$ & $0.89(0.79-1.01)$ \\
\hline Brother $^{\mathrm{C}}$ & & $9.61(7.24-12.75)$ & $9.37(7.04-12.48)$ \\
\hline \multicolumn{4}{|c|}{$\begin{array}{l}\text { a. Adjusted for calendar year, body mass index, region and conscription test } \\
\text { centre. } \\
\text { b. Reference category: high. } \\
\text { c. One or more brothers with specified diagnosis. }\end{array}$} \\
\hline
\end{tabular}

depression in low and medium (compared with high) cardiovascular fitness groups were $1.70(1.54-1.87)$ and $1.28(1.22-1.34)$ respectively. Taken together, the associations between cardiovascular fitness and later risk of serious depression were independent of muscle strength.

\section{Analyses of associations between cardiovascular fitness and serious depression at different follow-up times}

Three ICD versions were utilised during the long observation period. As this might affect results, we carried out separate analyses for each ICD system. These analyses showed similar associations between cardiovascular fitness and serious depression but with somewhat higher hazard ratios within the ICD-10 (data not shown). In order to see whether there were changes in the effect size with greater follow-up time, the cohort was divided into 10 -year periods with respect to conscription year and follow-up years (Table 7). Higher hazard ratios were observed closer to baseline within all four follow-up periods.

\section{Discussion}

\section{Main findings}

In this national cohort study, we demonstrate that lower cardiovascular fitness at age 18 predicted serious depression in adulthood. Associations were only slightly attenuated after controlling for a number of possible confounders including familial factors. The associations between cardiovascular fitness and later risk of serious depression were independent of muscle strength. The present study demonstrates that physical fitness in young adulthood has an impact on the long-term incidence of serious depression in males, extending findings from previous longitudinal studies involving older people with shorter followup times. ${ }^{10,11}$

Belonging to the low fitness group compared with the high fitness group was associated with a moderately increased risk of bipolar disorder. In contrast, belonging to the medium group compared with the high group was associated with a small but significantly reduced risk of bipolar disorder. One cross-sectional and prospective longitudinal study of adolescents followed for 4 years found the incidence of bipolar disorder to be increased 


\begin{tabular}{|c|c|c|c|}
\hline \multirow[b]{2}{*}{ Cardiovascular fitness ${ }^{a}$} & \multicolumn{3}{|c|}{ Hazard ratio $(95 \% \mathrm{Cl})$} \\
\hline & Age adjusted & Adjusted $^{\mathrm{b}}$ & Adjusted $^{\mathrm{C}}$ \\
\hline \multicolumn{4}{|l|}{ Stratified for low muscle strength } \\
\hline Cardiovascular fitness: Iow & $1.73(1.56-1.91)$ & $1.70(1.53-1.89)$ & $1.69(1.48-1.94)$ \\
\hline Cardiovascular fitness: medium & $1.32(1.24-1.42)$ & $1.27(1.19-1.36)$ & $1.20(1.10-1.31)$ \\
\hline \multicolumn{4}{|l|}{ Stratified for medium muscle strength } \\
\hline Cardiovascular fitness: Iow & $1.94(1.66-2.25)$ & $1.87(1.61-2.19)$ & $1.88(1.55-2.28)$ \\
\hline Cardiovascular fitness: medium & $1.37(1.29-1.45)$ & $1.33(1.25-1.42)$ & $1.35(1.25-1.45)$ \\
\hline \multicolumn{4}{|l|}{ Stratified for high muscle strength } \\
\hline Cardiovascular fitness: Iow & $1.72(1.37-2.18)$ & $1.70(1.34-2.16)$ & $1.71(1.29-2.25)$ \\
\hline Cardiovascular fitness: medium & $1.35(1.26-1.45)$ & $1.31(1.22-1.41)$ & $1.29(1.18-1.41)$ \\
\hline $\begin{array}{l}\text { a. Reference category: high. } \\
\text { b. Adjusted for body mass index (BMI) } \\
\text { c. Adjusted for calendar year, BMI, regi }\end{array}$ & education. & & \\
\hline
\end{tabular}

\begin{tabular}{|c|c|c|c|}
\hline \multirow[b]{2}{*}{ Cardiovascular fitness $^{a}$} & \multicolumn{3}{|c|}{ Hazard ratio $(95 \% \mathrm{Cl})$} \\
\hline & Age adjusted & Adjusted $^{\mathrm{b}}$ & Adjusted $^{\mathrm{C}}$ \\
\hline \multicolumn{4}{|c|}{ Stratified for low cardiovascular fitness } \\
\hline Muscle strength: low & $1.23(0.95-1.59)$ & $1.31(0.98-1.77)$ & $1.31(0.92-1.87)$ \\
\hline Muscle strength: medium & $1.17(0.92-1.50)$ & $1.24(0.94-1.62)$ & $1.18(0.86-1.62)$ \\
\hline \multicolumn{4}{|c|}{ Stratified for medium cardiovascular fitness } \\
\hline Muscle strength: low & $1.31(1.21-1.41)$ & $1.32(1.22-1.44)$ & $1.28(1.15-1.42)$ \\
\hline Muscle strength: medium & $1.06(1.00-1.13)$ & $1.08(1.02-1.15)$ & $1.07(0.99-1.15)$ \\
\hline \multicolumn{4}{|c|}{ Stratified for high cardiovascular fitness } \\
\hline Muscle strength: low & $1.30(1.15-1.48)$ & $1.31(1.15-1.50)$ & $1.31(1.11-1.54)$ \\
\hline Muscle strength: medium & $1.05(0.99-1.12)$ & $1.06(0.99-1.13)$ & $1.04(0.96-1.12)$ \\
\hline \multicolumn{4}{|c|}{$\begin{array}{l}\text { a. Reference category: high. } \\
\text { b. Adjusted for body mass index (BMI) and calendar year. } \\
\text { c. Adjusted for calendar year, BMI, region, conscription test centre and parental education. }\end{array}$} \\
\hline
\end{tabular}

\begin{tabular}{|c|c|c|c|c|c|c|c|c|}
\hline \multirow[b]{3}{*}{ Conscript year } & \multicolumn{8}{|c|}{ Follow-up period } \\
\hline & \multicolumn{2}{|c|}{ 1969-1978 (ICD-8) } & \multicolumn{2}{|c|}{ 1979-1988 (ICD-8/9) } & \multicolumn{2}{|c|}{ 1989-1998 (ICD-9/10) } & \multicolumn{2}{|c|}{ 1999-2008 (ICD-10) } \\
\hline & $n$ & $\mathrm{HR}(95 \% \mathrm{Cl})$ & $n$ & HR $(95 \% \mathrm{Cl})$ & $n$ & $\mathrm{HR}(95 \% \mathrm{Cl})$ & $n$ & HR $(95 \% \mathrm{Cl})$ \\
\hline 1968-1977 & 641 & & 1196 & & 1622 & & 2288 & \\
\hline Cardiovascular fitness: ${ }^{\mathrm{b}}$ low & & $2.37(1.45-3.87)$ & & $1.93(1.38-2.70)$ & & $1.52(1.12-2.06)$ & & $1.36(1.06-1.74)$ \\
\hline Cardiovascular fitness: ${ }^{\mathrm{b}}$ medium & & $1.70(1.31-2.22)$ & & $1.19(1.00-1.42)$ & & $1.22(1.06-1.41)$ & & $1.12(1.00-1.26)$ \\
\hline 1978-1987 & & & 538 & & 1292 & & 2250 & \\
\hline Cardiovascular fitness: ${ }^{\mathrm{b}}$ low & & & & $2.07(1.34-3.19)$ & & $1.56(1.18-2.07)$ & & $1.88(1.54-2.30)$ \\
\hline Cardiovascular fitness: ${ }^{b}$ medium & & & & $1.02(0.80-1.30)$ & & $1.25(1.09-1.44)$ & & $1.28(1.15-1.43)$ \\
\hline 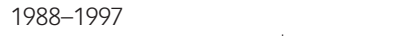 & & & & & 600 & & 1761 & \\
\hline Cardiovascular fitness: ${ }^{\text {b low }}$ & & & & & & $2.07(1.14-3.77)$ & & $3.35(2.56-4.39)$ \\
\hline Cardiovascular fitness: ${ }^{b}$ medium & & & & & & $1.63(1.30-2.04)$ & & $1.70(1.50-1.93)$ \\
\hline
\end{tabular}

among those with higher physical activity at baseline. ${ }^{25}$ Associations of low cardiovascular fitness and bipolar disorder did not remain in the subanalyses within full-brother pairs. This may reflect the greater influence of genetic factors on bipolar disorder compared with depression. ${ }^{26}$ Although the positive impact of physical exercise in clinical populations makes it attractive as an adjunct treatment also for bipolar disorder, ${ }^{27,28}$ our findings cannot support the theory that cardiovascular fitness can reduce risk for the development of bipolar disorder. The incidence and prevalence of unipolar depression is influenced by cultural factors and other determinants to a larger extent than bipolar disorder, and unipolar depression might thus be more amenable to preventive interventions. ${ }^{29}$

\section{Strengths and limitations}

Important strengths of this study are the size $(>1.1$ million individuals), the prospective population-based design, the long follow-up time and the use of an objective measure of 
cardiovascular fitness. The Swedish National Hospital Discharge Register enables us to cover virtually all in-patient care for affective disorders during 40 years. An additional strength of this study is the reliance on psychologists and medical doctors for the baseline measure of mental health, allowing us to exclude individuals with pre-existing psychiatric disorders or symptoms. However, there are also drawbacks to the current design. Although the conscripts received extensive examinations by psychologists, we only have information about clinical symptoms or disorders and not data in the form of comparable depression and anxiety scales, which could help in defining if subsyndromal affective problems affect the associations in some way. There are also other possible confounders that may increase risk for both poor fitness and depression, that we were not able to control for, such as personality, smoking and low self-esteem.

The incidence of serious depression could be somewhat underestimated as some participants with only primary healthcare/ out-patient psychiatric contact would not be included in the hospital register. On the other hand, we may also have captured some individuals who required admission to hospital despite only moderate depression. However, restricting our definition of cases to only individuals requiring in-patient care by psychiatric specialists should increase the diagnostic reliability.

Another limitation is that the Hospital Discharge Register only includes data at diagnosis level, whereas differences at the symptom level could also be important. Cardiovascular fitness may correlate differentially with individual depression symptoms. Future studies with comparisons at the symptom level may stimulate a more cognisant discussion of how specific brain regions might be influenced by physical fitness.

An open question is how those who seek in-patient care differ from those who do not, and how this would affect the results. A recent Swedish study showed that those who do not seek care for mental health problems are more likely to be men, to have lower education, lower social support and feelings of shame that would prevent them from seeking care. ${ }^{30}$ By restricting the definition of cases to individuals with in-patient care, we may have selected for individuals with higher education and a better social network. To mitigate this potential bias, we controlled for parental education, which did not alter results in a significant manner. Thus, it is unlikely that the associations we examined would be particularly sensitive to this selection bias. Highly educated parents may also encourage their children to exercise more and also encourage participation in activities that may improve cognitive and psychological performance. This is a classic example of a potential gene-environment correlation. ${ }^{31}$ Therefore, we included parental educational level as a confounder and performed subgroup analyses. Low cardiovascular fitness at age 18 strongly increased risk of later depression, regardless of level of parental education.

An almost general observation, independent of nationality or culture, is the twofold greater prevalence of major depression in women (10-25\% lifetime prevalence) compared with men (5-12\% lifetime prevalence). ${ }^{32}$ Explanations may involve behavioural and hormonal differences, including effects of childbirth and differing psychosocial stressors for women and for men. In the present study, as only males were examined, we cannot extrapolate our findings to the female population.

\section{Interpretation}

Our findings are not explanatory with respect to causal chains leading to the onset of depression. Depression is an aetiologically complex disorder influenced by many factors from multiple domains. To ascertain a risk factor, it must precede onset of, and correlate with the disorder. This is achieved using a longitudinal prospective study, such as the current study, where the association between the risk factor and onset can be because of either a causal effect of the risk factor, and/or to any factor(s) associated with that risk factor. ${ }^{33}$ Childhood risk factors for depression include genetic liability, poor parenting, traumatic experiences, poor self-esteem and predisposing personality traits, ${ }^{34}$ and they represent a possibility for reverse causality as these factors may affect fitness levels at conscription. In order to assure that low physical fitness precedes onset of depression, we excluded individuals that fulfilled criteria for previous or ongoing mental and behavioural disorders and symptoms as well as individuals with previously registered in-patient affective disorder diagnoses.

The association between cardiovascular fitness and serious depression decreased with longer observations periods, although it remained significant. Other studies also show that risk factors for depression have the strongest impact close to onset and in order to retain any association with depression in adulthood, the risk factor has to be assessed after adolescence and not before. ${ }^{35}$ However, whether low physical activity simply reflects the presence of early undetected depressive symptoms or whether it actually affects onset and clinical course of adult depression need further investigation.

By performing subanalyses within full-brother pairs, many of the early childhood risk factors could be accounted for, including genetics and parental treatment and upbringing. If the association between cardiovascular fitness and depression was entirely explained by genetic and rearing conditions, the association would be substantially reduced or even disappear within full-brother pairs. We assessed potential effects of familial factors, by performing separate Cox regression analyses among brother pairs. However, in contrast to bipolar disorder the association between cardiovascular fitness and depression remained strong. Interestingly, hazard risk figures were very similar in sib-controlled and in standard analyses. This argues against a substantial genetic or family environmental correlation between cardiovascular fitness and risk of severe depression. This issue has been addressed in a twin study. ${ }^{12}$ De Moor and colleagues examined self-reports of exercise activity and depressive symptoms and found only modest genetic correlations $(-0.24$ and -0.20 for cross-sectional and longitudinal analyses, respectively, in men). They reported no association between exercise and depressive symptoms within pair analyses of monozygotic twins. Our data, which are based on a much better powered sample, support the hypothesis that objective measures of cardiovascular fitness are indeed associated with major depression. However, we have studied the association among full brothers and not monozygotic twins.

There are other biases. For example, participants with equal levels of depressive symptoms may more likely be admitted to hospital for depression if they have previously had other medical consequences of poor cardiovascular fitness before the onset of depression, such as cardiovascular disease. However, findings remained robust after the exclusion of men with circulatory system disease, which speaks against a significant confounding effect.

Cardiovascular disease and depression have a high comorbidity rate. ${ }^{36}$ Atherosclerosis, the underlying process leading to vascular events, has been associated with depression. ${ }^{37}$ The 'vascular depression hypothesis' proposes that vascular factors precede the onset of depression in older adults. Patients with late-life depression exhibit characteristic white matter brain lesions and large artery endothelial dysfunction. ${ }^{38,39}$ Genetic factors, cardiovascular risk factors or diet have been suggested as disease modulators for both cardiovascular disease and depression, ${ }^{40-42}$ 
and in a recent study depressive predictor variables correlated highly with vascular risk factor severity. ${ }^{43}$ An important cardiovascular risk factor is low cardiovascular fitness. Indeed, we found that low cardiovascular fitness at age 18 was a risk factor for serious depression, even 31-40 years later. However, we already observed an association between cardiovascular fitness and risk of depression at a young age (i.e. within 1-10 years), and we therefore propose that depression in young adulthood may also have a cardiovascular component. If so, this would perhaps be classified as the subclinical cardiovascular hypothesis (i.e. no visible changes in white matter or endothelium). Depression, brain lesions and endothelial dysfunction may all result from an underlying biological substrate related to lack of cardiovascular exercise.

\section{Implications}

Lower cardiovascular fitness at age 18 was associated with an increased risk of serious depression in adulthood. These results strengthen the theory of a cardiovascular contribution to the aetiology of depression. Although the results in the present population-based prospective study are compelling, a number of confounders could not be measured and intervention studies are needed to determine whether physical exercise in young adulthood can prevent future onset of depression. It would be of great interest to target specific high-risk groups for developing depression for intervention with cardiovascular training. Mechanistic studies on mediating factors of cardiovascular fitness at the biochemical level could provide insights into future preventive and treatment strategies for affective disorders.

Maria A. I. Åberg, MD, PhD, Center for Brain Repair and Rehabilitation, Institute fo Neuroscience and Physiology and Department of Primary Health Care, Institute of Medicine, Sahlgrenska Academy, University of Gothenburg, Gothenburg, Sweden; Margda Waern, MD, PhD, Department of Psychiatry and Neurochemistry, Neuropsychiatric Epidemiology Unit, Institute of Neuroscience and Physiology, Sahlgrenska Academy, University of Gothenburg, Gothenburg, Sweden; Jenny Nyberg, PhD, Center for Brain Repair and Rehabilitation, Institute for Neuroscience Nyberg, PhD, Center for Brain Repair and Rehabilitation, Institute for Neuroscienc
and Physiology, Sahlgrenska Academy, University of Gothenburg, Gothenburg, Sweden; Nancy L. Pedersen, PhD, Department of Psychology, University of Southern California, Los Angeles, California, USA, and Department of Medical Epidemiology and Biostatistics at Karolinska Institute, Stockholm, Sweden; Ylva Bergh, MD, Department of Primary Health Care, Institute of Medicine, Sahlgrenska Academy, University of Gothenburg, Gothenburg, Sweden; N. David Åberg, MD, PhD, Laboratory of Experimental Endocrinology, Institute of Medicine, Sahlgrenska Academy, University of Gothenburg, Gothenburg, Sweden; Michael Nilsson, MD, PhD, H. Georg Kuhn, PhD, Center for Brain Repair and Rehabilitation, Institute for Neuroscience and Physiology, Sahlgrenska Academy, University of Gothenburg, Gothenburg, Sweden; Kjell Torén, MD, PhD, Occupational and Environmental Medicine, Institute of Medicine, Sahlgrenska Academy, University of Gothenburg, Gothenburg, Sweden

Correspondence: $\mathrm{H}$. Georg Kuhn, PhD, Institute for Neuroscience and Physiology, University of Gothenburg, Medicinaregatan 11, Box 432, S-40530 Gothenburg, Sweden. Email: Georg.Kuhn@neuro.gu.se

First received 21 Sep 2011, final revision 17 Feb 2012, accepted 3 Apr 2012

\section{Funding}

This study was supported by grants from the Märtha Lundqvists Stiftelse, the Swedish Re search Council for Worklife and Social Science (FAS) and the Swedish government under the LUA/ALF agreement for biomedical research.

\section{References}

1 Kubesch S, Bretschneider V, Freudenmann R, Weidenhammer N, Lehmann $M$, Spitzer $M$, et al. Aerobic endurance exercise improves executive functions in depressed patients. J Clin Psychiatry 2003; 64: 1005-12.

2 Hoffman BM, Babyak MA, Craighead WE, Sherwood A, Doraiswamy PM, Coons MJ, et al. Exercise and pharmacotherapy in patients with major depression: one-year follow-up of the SMILE study. Psychosom Med 2011; 73: $127-33$.
3 Calabrese F, Molteni R, Racagni G, Riva MA. Neuronal plasticity: a link between stress and mood disorders. Psychoneuroendocrinology 2009; 34 (suppl 1): S208-16.

4 Duman $\mathrm{CH}$, Schlesinger L, Russell DS, Duman RS. Voluntary exercise produces antidepressant and anxiolytic behavioral effects in mice. Brain Res 2008; 1199: 148-58

5 Alsuwaidan MT, Kucyi A, Law CW, McIntyre RS. Exercise and bipolar disorder: a review of neurobiological mediators. Neuromolecular Med 2009; 11: $328-36$

6 De Moor MH, Beem AL, Stubbe JH, Boomsma DI, De Geus EJ. Regular exercise, anxiety, depression and personality: a population-based study. Prev Med 2006; 42: 273-9.

7 Harvey SB, Hotopf M, Øverland S, Mykletun A. Physical activity and common mental disorders. Br J Psychiatry 2010; 197: 357-64.

8 Sund AM, Larsson B, Wichstrom L. Role of physical and sedentary activities in the development of depressive symptoms in early adolescence. Soc Psychiatry Psychiatr Epidemiol 2011; 46: 431-41.

9 O'Farrell A, Flanagan E, Bedford D, James D, Howell F. Factors associated with self-reported depression and self-esteem among school-going adolescents from a geographically defined region in Ireland. Ir J Med SCi 2005; 174: 17-22.

10 Farmer ME, Locke BZ, Moscicki EK, Dannenberg AL, Larson DB, Radloff LS. Physical activity and depressive symptoms: the NHANES I epidemiologic follow-up study. Am J Epidemiol 1988; 128: 1340-51.

11 Strawbridge WJ, Deleger S, Roberts RE, Kaplan GA. Physical activity reduces the risk of subsequent depression for older adults. Am J Epidemiol 2002; 156: $328-34$

12 De Moor MH, Boomsma DI, Stubbe JH, Willemsen G, de Geus EJ. Testing causality in the association between regular exercise and symptoms of anxiety and depression. Arch Gen Psychiatry 2008; 65: 897-905.

13 Rothon C, Edwards P, Bhui K, Viner RM, Taylor S, Stansfeld SA. Physical activity and depressive symptoms in adolescents: a prospective study. BMC Med 2010; 8: 32

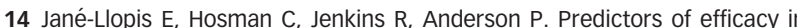
depression prevention programmes. Meta-analysis. Br J Psychiatry 2003 183: 384-97.

15 Aberg MA, Pedersen NL, Toren K, Svartengren M, Backstrand B, Johnsson T, et al. Cardiovascular fitness is associated with cognition in young adulthood. Proc Natl Acad Sci U S A 2009; 106: 20906-11.

16 Castelli DM, Hillman $\mathrm{CH}$, Buck SM, Erwin HE. Physical fitness and academic achievement in third- and fifth-grade students. J Sport Exerc Psychol 2007: 29: $239-52$.

17 Nordesjö LO, Schéle R. Validity of an ergometer cycle test and measures of isometric muscle strength when prediction some aspects of military performance. Swed J Def Med 1974; 10: 11-23.

18 Glassford RG, Baycroft GH, Sedgwick AW, Macnab RB. Comparison of maximal oxygen uptake values determined by predicted and actual methods. J Appl Physiol 1965; 20: 509-13.

19 Nordesjö LO. A comparison between the Thornvall maximal ergometer test, submaximal ergometer tests and maximal oxygen uptake. Swed J Def Med 1974; 10: 3-10

20 World Health Organization. International Statistical Classification of Diseases and Related Health Problems (ICD-8). WHO, 1967.

21 World Health Organization. International Statistical Classification of Diseases and Related Health Problems (ICD-9). WHO, 1978.

22 World Health Organization. The ICD-10 Classification of Mental and Behavioural Disorders: Clinical Descriptions and Diagnostic Guidelines. WHO, 1992.

23 Calkin C, van de Velde C, Ruzickova M, Slaney C, Garnham J, Hajek T, et al. Can body mass index help predict outcome in patients with bipolar disorder? Bipolar Disord 2009: 11: 650-6.

24 Vogelzangs N, Kritchevsky SB, Beekman AT, Brenes GA, Newman AB, Satterfield $\mathrm{S}$, et al. Obesity and onset of significant depressive symptoms: results from a prospective community-based cohort study of older men and women. J Clin Psychiatry 2010; 71: 391-9.

25 Strohle A, Hofler M, Pfister H, Muller AG, Hoyer J, Wittchen HU, et al. Physical activity and prevalence and incidence of mental disorders in adolescents and young adults. Psychol Med 2007; 37: 1657-66.

26 Li X, Sundquist K, Hemminki K, Sundquist J. Familial risks for depression among siblings based on hospitalizations in Sweden. Psychiatr Genet 2008; 18: $80-4$.

$27 \mathrm{Ng} F$, Dodd S, Berk M. The effects of physical activity in the acute treatment of bipolar disorder: a pilot study. J Affect Disord 2007; 101: 259-62.

28 Edenfield TM. Exercise and mood: exploring the role of exercise in regulating stress reactivity in bipolar disorder. Diss Abstr Int B Sci Eng 2008; 68: 5566. 
29 Weissman MM, Bland RC, Canino GJ, Faravelli C, Greenwald S, Hwu HG, et al Cross-national epidemiology of major depression and bipolar disorder. JAMA 1996; 276: 293-9.

30 Forsell $Y$. The pathway to meeting need for mental health services in Sweden. Psychiatr Serv 2006; 57: 114-9.

31 Plomin R, DeFries JC, McClearn GE, McGuffin P. Behavioral Genetics (4th edn). Worth Publishers, 2001

32 Sadock BJ, Sadock VA. Mood disorders. In Kaplan and Sadock's Synopsis of Psychiatry: Behavioral Sciences/Clinical Psychiatry (9th edn) (eds BJ Sadock, VA Sadock): 534-90. Lippincott Williams and Wilkins, 2003.

33 Kraemer HC. Epidemiological methods: about time. Int J Environ Res Public Health 2010; 7: 29-45.

34 Kendler KS, Gardner CO, Prescott CA. Toward a comprehensive developmental model for major depression in men. Am J Psychiatry 2006; 163: $115-24$.

35 Shanahan L, Copeland WE, Costello EJ, Angold A. Child-, adolescent- and young adult-onset depressions: differential risk factors in development? Psychol Med 2011; 6: 1-10.

36 Carbone JR, Gorman JM, Goodman J, Willems MB. Mood disorders and the heart. In Medical and Psychiatric Comorbidity Over the Course of Life (ed WW Eaton): 97-127. American Psychiatric Publishing, 2006.
37 Bus BA, Marijnissen RM, Holewijn S, Franke B, Purandare N, de Graaf J, et al Depressive symptom clusters are differentially associated with atherosclerotic disease. Psychol Med 2011; 41: 1419-28.

38 Dalby RB, Chakravarty MM, Ahdidan J, Sorensen L, Frandsen J, Jonsdottir KY, et al. Localization of white-matter lesions and effect of vascular risk factors in late-onset major depression. Psychol Med 2010; 40: 1389-99.

39 Greenstein AS, Paranthaman R, Burns A, Jackson A, Malik RA, Baldwin RC et al. Cerebrovascular damage in late-life depression is associated with structural and functional abnormalities of subcutaneous small arteries. Hypertension 2010; 56: 734-40.

40 Kendler KS, Gardner CO, Fiske A, Gatz M. Major depression and coronary artery disease in the Swedish twin registry: phenotypic, genetic, and environmental sources of comorbidity. Arch Gen Psychiatry 2009; 66: 857-63.

41 Bondy B. Common genetic factors for depression and cardiovascular disease Dialogues Clin Neurosci 2007; 9: 19-28.

42 Baghai TC, Varallo-Bedarida G, Born C, Hafner S, Schule C, Eser D, et al Major depressive disorder is associated with cardiovascular risk factors and low Omega-3 index. J Clin Psychiatry 2011; 72: 1242-7.

43 Sheline YI, Pieper CF, Barch DM, Welsh-Bohmer K, McKinstry RC, MacFall JR, et al. Support for the vascular depression hypothesis in late-life depression: results of a 2-site, prospective, antidepressant treatment trial. Arch Gen Psychiatry 2010; 67: 277-85.

\section{psychiatry in literature}

\section{Ophelia in Hamlet by William Shakespeare}

\section{Jo Richards}

Hamlet continues to fascinate contemporary actors and audiences. The dramatic possibilities which arise from the main character deciding to 'put an antic disposition on', i.e. pretend to be mad, provides an intriguing study for psychiatrists.

The play begins with Hamlet meeting his deceased father's ghost. The ghost reveals how he was murdered by his own brother who has now married his widow, Hamlet's mother. Following the ghost's visitation Hamlet's emotional and behavioural turmoil escalates. He rejects fair Ophelia and accidentally kills her father Polonius. Audiences understand how Hamlet wrestles with continuing suicidal and homicidal urges as the plot progresses. Hamlet's soliloquies during Act III and IV reveal the turbulent emotional and intellectual inner world of a desperate man. We understand Hamlet's destructive urges, 'Now could I drink hot blood'. We hear and see his most private discussions with himself about whether revenge is justified. By Act $\mathrm{V}$ we know that Hamlet is himself prepared for death, 'The readiness is all'.

Following Hamlet's famous misogynistic attack on Ophelia in Act III, 'Get thee to a nunnery. . . ', Shakespeare gives the latter a single soliloquy. She eloquently comments on Hamlet's seeming mental breakdown, 'O, what a noble mind is here o'erthrown' and her own sadness, 'And I, of ladies most deject and wretched/That sucked the honey of his music vows'. However, as Ophelia's grief increases following her father's death, so does her passivity. This contrasts with Hamlets' active attempts to resolve his internal and real life crises. Audiences see Ophelia unravelling mentally through the eyes of the other characters during Act IV such as Claudius, ' . . . poor Ophelia/Divided from herself and her fair judgement'. We and they attempt to decipher the meaning behind her speech and behaviour while she ceases to engage directly with us. Instead she speaks disjointedly and sings, seeming to allude to death, 'They bore him barefaced on the brier/Hey non nony. . . '. Later, Gertrude describes how Ophelia subsequently drowns while picking wildflowers. Gertrude's account of Ophelia falling in the water portrays the latter as helpless and disconnected from real danger. 'Her clothes spread wide ... they bore her up ... she chanted snatches of old tunes/As one incapable of her own distress... Till that her garments, heavy with their drink/Pulled the poor wretch from her melodious lay/To muddy death'. Ophelia's death can be interpreted as suicide. There is uncertainty about this in the play, recognisable as equivalent to a present day coroner's open verdict.

Considering Hamlet and Ophelia together, we observe different responses to loss and abandonment. It is Shakespeare's skil in creating the characters which binds us into this old tragedy still. He does this through their clever use of language, their relationships with other characters in the play and interaction with audiences. We love to listen to the momentous soliloquies of the Prince of Denmark, alone on the stage with us but each time seeking variation, a new interpretation. But the vulnerable Ophelia has also become iconic. Ophelia's transformation from an eloquent to an elusive and disengaged character charts her particular dispossession.

The quotations are from the 1996 Penguin Books edition (ed. T. J. B. Spence). 